\section{BMJ Open} Ophthalmology

\title{
Association between serum soluble fms- like tyrosine kinase- 1 and the central choroidal thickness during pregnancy: a prospective study
}

Takafumi Suzuki (D) , ${ }^{1,2}$ Tatsuya Inoue, ${ }^{2,3}$ Nozomi Igarashi, ${ }^{2}$ Chisato Agata, ${ }^{2}$ Atsushi Okubo, ${ }^{2}$ Takeshi Nagamatsu, ${ }^{4}$ Takayuki Iriyama, ${ }^{4}$ Maiko Maruyama-Inoue, ${ }^{3}$ Yasuo Yanagi, ${ }^{3}$ Kazuaki Kadonosono, ${ }^{3}$ Ryo Asaoka (D) , ${ }^{2}$ Keiko Azuma, ${ }^{2}$ Ryo Obata (1) ${ }^{2}$

To cite: Suzuki T, Inoue T, Igarashi N, et al. Association between serum soluble fmslike tyrosine kinase- 1 and the central choroidal thickness during pregnancy: a prospective study. BMJ Open Ophthalmology 2021;6:e000888. doi:10.1136/ bmjophth-2021-000888

Received 31 August 2021 Accepted 23 October 2021

Check for updates

(C) Author(s) (or their employer(s)) 2021. Re-use permitted under CC BY-NC. No commercial re-use. See rights and permissions. Published by BMJ.

${ }^{1}$ Department of Ophthalmology, Shinseikai Toyama Hospital, Imizu-gun, Toyama, Japan

${ }^{2}$ Department of Ophthalmology, The University of Tokyo Hospital, Bunkyo-ku, Tokyo, Japan ${ }^{3}$ Department of Ophthalmology and Micro-Technology, Yokohama City University Graduate School of Medicine, Yokohama, Japan

${ }^{4}$ Department of Obstetrics and Gynecology, The University of Tokyo Hospital, Bunkyo-ku, Tokyo, Japan

Correspondence to Dr Tatsuya Inoue; inouet-tky@ umin.ac.jp

\section{ABSTRACT}

Objective To investigate the association between serum soluble fms-like tyrosine kinase-1 (sFlt-1) and the choroidal structure during the pregnancy.

Methods and analysis This prospective study enrolled 24 eyes of 12 consecutive healthy pregnant women. Each participant was evaluated during the first, second and third trimester of pregnancy and the postpartum period. The central choroidal thickness (CCT) and Choroidal Vascular Index (CVI) were measured using enhanced depth-imaging optical coherence tomography. Moreover, serum sFlt-1 and vascular endothelial growth factor (VEGF) concentrations were measured manually using ELISA. The association between choroidal structural parameters and serum sFlt-1/ VEGF was analysed using a linear mixed model.

Results The CCT was significantly thicker in the second and third trimester than the postpartum period $(p<0.05)$. In contrast, there was no significant difference in the CVI throughout pregnancy or the postpartum period. Serum sFlt-1 levels were significantly higher during pregnancy than the postpartum period $(p<0.05)$, whereas the serum VEGF levels were significantly lower during pregnancy than the postpartum period $(p<0.05)$. Furthermore, serum sFlt-1 levels were significantly associated with CCT throughout pregnancy $(p=0.011$, linear mixed model). On the contrary, sFlt-1 levels was not associated with the CVI during the pregnancy.

Conclusion Our results suggest that serum sFlt-1 might have an influence on the choroidal thickness during the pregnancy.

\section{INTRODUCTION}

Physiological changes, including vascular, endocrine and metabolic changes, occur in the eyeball during the pregnancy. ${ }^{1}$ The dramatic changes in the ocular blood flow during pregnancy have been reported to cause various retinal and choroidal diseases, such as central serous chorioretinopathy (CSC). ${ }^{23}$

CSC is one of the most common causes of visual impairment during pregnancy.

\section{Key messages}

What is already known about this subject?

$>$ In the field of obstetrics, serum vascular endothelial growth factor (VEGF) and soluble fms-like tyrosine kinase-1 (sFlt-1) are known to be associated with pregnancy, miscarriage, gestational hypertension and pre-eclampsia.

VEGF has been implicated as a possible cause of choroidal vascular hyperpermeability in eyes with central serous chorioretinopathy (CSC).

- The dramatic changes in the ocular blood flow during pregnancy have been reported to cause various retinal and choroidal diseases, such as CSC.

\section{What are the new findings?}

- We investigated the choroidal structure in detail, including the central choroidal thickness and Choroidal Vascular Index (CVI), throughout pregnancy. We also investigated the factors affecting any choroida structural changes.

- The choroidal thickness was significantly higher in the second and third trimester of pregnancy than in the postpartum period; however, this was not the case with the CVI during pregnancy. Moreover serum sFlt-1 was significantly associated with choroidal thickness during pregnancy. Taken together sFlt-1 has a physiological effect on the choroida structure during the pregnancy.

\section{How might these results change the focus of research or clinical practice? \\ - It is possible that measurement of choroidal struc- ture may be useful for predicting the pregnancy- related diseases such as pre-eclampsia.}

Although the exact mechanism of this phenomenon still remains elusive, it may be related to various hormonal changes, such as increased levels of glucocorticoid during pregnancy, since systemic steroid intake is a known cause of CSC. ${ }^{4}$ Further, vascular endothelial growth factor (VEGF) has been 
implicated as a possible cause of choroidal vascular hyperpermeability in eyes with CSC. ${ }^{5}$

In the field of obstetrics, serum VEGF and soluble fmslike tyrosine kinase-1 (sFlt-1), a truncated form of the VEGF receptor 1, are known to be associated with pregnancy and miscarriage. ${ }^{6}$ Moreover, a previous study has revealed that elevated serum sFlit-1 and decreased serum VEGF levels were associated with gestational hypertension and pre-eclampsia. ${ }^{7}$

Recent several studies suggested that binarisation method of optical coherence tomography (OCT) image is a promising way to reveal the choroidal structure in many retinal diseases, such as age-related macular degeneration and CSC. Sonoda et al have proposed a method to discriminate choroidal luminal area using binarised OCT images. ${ }^{89}$ Histopathological findings are useful for understanding of choroidal architecture, however, there are artefacts subjacent to tissue fixation. In comparison with histopathological examination, OCT binarisation method provides more reproducible in vivo information without altering vascular diameter. Several reports have suggested the possibility of choroidal changes during pregnancy by focusing on the central choroidal thickness (CCT). Using the binarisation technique, we recently investigated the choroidal structure during the first trimester of pregnancy. ${ }^{10}$ Our results showed that in the first trimester of pregnancy, the Choroidal Vascular Index (CVI) was higher compared with that of the nonpregnant women in the control group. In contrast, the CCT was not significantly different between the pregnant and control groups. However, in our previous cross-sectional study, changes in the choroidal structure throughout pregnancy were not investigated.

To the best of our knowledge, there have been no reports investigating the choroidal structure in detail, including the CVI, throughout pregnancy and postpartum periods, and the factors affecting any choroidal structural changes. Therefore, we aimed to investigate the choroidal structural changes in the first, second and third trimesters during pregnancy compared with those in the postpartum period, and to analyse the association between serum sFlt-1 level and the choroidal structure.

\section{MATERIALS AND METHODS}

Written informed consent was obtained from all participants, and our study was conducted in accordance with the Declaration of Helsinki. Patients or the public were involved in the design, or conduct, or reporting, or dissemination plans of our research. This prospective study enrolled pregnant women and data were collected from the first trimester of pregnancy. The participants with some complications of pregnancy such as intrauterine growth restriction or pre-eclampsia were excluded in this study.

Ophthalmic examinations were performed in conjunction with the obstetric consultation at four points: in the first trimester (10-14 weeks), second trimester (19-24 weeks), third trimester (26-37 weeks) and postpartum period (2-15 weeks after delivery). Each participant underwent a comprehensive ophthalmological examination, including visual acuity, axial length (AL) measured by partial coherence interferometry (IOL Master, Tomey OA-2000, V.5.4.4.0006; Tomey, Nagoya, Japan), and refractive error measured by autorefraction (KR-8900 V.1.07; Topcon, Tokyo, Japan). To measure the CCT and CVI, enhanced depth-imaging OCT images were obtained using spectral domain OCT (Spectralis; Heidelberg Engineering, Heidelberg, Germany) at each visit. Binarised images were obtained using the Niblack method (ImageJ software), as previously described. ${ }^{8}$ In brief, an image of one horizontal scan passing through the fovea was selected and then converted into eight bits, and the Niblack auto-local threshold was applied to binarise the images and separate the choroidal luminal and stromal areas. After binarisation, the total choroidal area, luminal area and CVI were calculated for each eye.

\section{Measurement of serum VEGF and serum sFlt-1}

The levels of VEGF and sFlt-1 in serum samples were measured using human VEGF (DVE00) and human sFlt-1 Quantikine ELISA Kit (DVR100C) (R\&D Systems, Minneapolis, Minnesota, USA), following the manufacturer's protocol. Owing to the skewed distribution of the raw values, serum VEGF and sFlt-1values were normalised through logarithmic transformation $(\log (\mathrm{VEGF}), \log (\mathrm{s}-$ Flt-1)). The lower limit of detection was $5.0 \mathrm{pg} / \mathrm{mL}$ in human VEGF Quantikine ELISA Kit, therefore the serum VEGF values measuring less than $5.0 \mathrm{pg} / \mathrm{mL}$ were set to $5.0 \mathrm{pg} / \mathrm{mL}$ for log-transformation.

\section{Statistical analyses}

A linear mixed model and Tukey's multiple comparison test were used to compare the values of CCT, CVI, $\log$ (VEGF) and $\log$ (sFlt-1) across the four assessment times (first, second, third trimester and post partum). By using a linear mixed model, measurements were nested within subjects and were dependent on each other. Thereafter, Tukey's multiple comparison test was performed for each variable comparison.

The associations between (1) $\log (\mathrm{sFlt}-1)$ and $\log$ (VEGF), (2) $\log$ (sFlt-1) and CCT, and (3) $\log ($ sFlt-1) and CVI were investigated using the linear mixed model. To determine the best explanatory variables for the CCT or CVI throughout the pregnancy, the model selection was performed using second-order bias-corrected Akaike information criterion index (AICc). Among age, AL, systolic blood pressure (SBP), diastolic blood pressure (DBP) and $\log (\mathrm{sFlt}-1)$, the optimal model for the CCT or CVI was identified from $2^{5}$ patterns. All statistical analyses were performed using the statistical programming language 'R' (V.3.1.3; The R Foundation for Statistical Computing, Vienna, Austria). 


\section{RESULTS}

Demographic data of the participants can be seen in table 1 . Twenty-four eyes of 12 consecutive healthy pregnant women were enrolled in this study. The CCT ratios to postpartum period were $1.04 \pm 0.09,1.10 \pm 0.13$ and $1.09 \pm 0.12$ in the first, second and third trimesters of pregnancy, respectively (figure 1A). These CCT ratios were significantly larger than $1.00 \quad(\mathrm{p}=0.0043$ in the second trimester, $\mathrm{p}=0.0086$ in the third trimester, linear mixed model and Tukey multiple comparisons test).

\begin{tabular}{|c|c|c|}
\hline & Median & IQR \\
\hline No of eyes & 24 & - \\
\hline Age (years) & 38 & $36.5-40.0$ \\
\hline \multicolumn{3}{|l|}{$\mathrm{CCT}(\mu \mathrm{m})$} \\
\hline First trimester & 292.0 & $239.8-369.2$ \\
\hline Second trimester & 307.5 & $253.8-382.2$ \\
\hline Third trimester & 302.0 & $261.2-383.5$ \\
\hline Postpartum & 276.5 & $211.5-365.0$ \\
\hline \multicolumn{3}{|l|}{ CVI (\%) } \\
\hline First trimester & 65.2 & $63.3-66.3$ \\
\hline Second trimester & 63.3 & $62.3-65.7$ \\
\hline Third trimester & 63.4 & $61.5-66.9$ \\
\hline Post partum & 64.2 & $63.0-65.3$ \\
\hline \multicolumn{3}{|l|}{$\mathrm{SBP}(\mathrm{mm} \mathrm{Hg})$} \\
\hline First trimester & 117.0 & $101.8-118.0$ \\
\hline Second trimester & 108.0 & $100.8-120.2$ \\
\hline Third trimester & 105.0 & $103.2-113.5$ \\
\hline Post partum & 113.5 & $105.2-119.2$ \\
\hline \multicolumn{3}{|l|}{ DBP (mm Hg) } \\
\hline First trimester & 71.0 & $64.25-75.25$ \\
\hline Second trimester & 64.0 & $57.75-72.25$ \\
\hline Third trimester & 64.0 & 59.5-68.5 \\
\hline Postpartum & 69.5 & $63.75-73.25$ \\
\hline \multicolumn{3}{|l|}{ Log(VEGF) (pg/mL) } \\
\hline First trimester & 0.70 & $0.70-0.70$ \\
\hline Second trimester & 0.70 & $0.70-0.75$ \\
\hline Third trimester & 0.70 & $0.70-0.75$ \\
\hline Post partum & 2.15 & $1.98-2.27$ \\
\hline \multicolumn{3}{|l|}{ Log(sFlt-1) (pg/mL) } \\
\hline First trimester & 3.56 & $3.55-3.57$ \\
\hline Second trimester & 3.55 & $3.52-3.55$ \\
\hline Third trimester & 3.56 & $3.55-3.57$ \\
\hline Post partum & 2.56 & $2.50-2.60$ \\
\hline
\end{tabular}

$\mathrm{BP}$, blood pressure; CCT, central choroidal thickness; CVI, Choroidal Vascular Index; DBP, diastolic blood pressure; IQR, interquartile range; SBP, systolic blood pressure; sFlt-1, soluble fms-like tyrosine kinase-1; VEGF, vascular endothelial growth factor.
In contrast, the CVIs ratios in the postpartum period were $1.02 \pm 0.06,1.01 \pm 0.06$ and $1.01 \pm 0.05$ in the first, second and third trimesters of pregnancy, respectively (figure 1B). These CVI ratios were not significantly different from 1.00 ( $p>0.05$, linear mixed model and Tukey multiple comparison test).

$\log (\mathrm{sFlt}-1)$ was significantly greater during pregnancy than the postpartum period (figure $2 \mathrm{~A}, \mathrm{p}<0.001$, linear mixed model and Tukey multiple comparisons test).

In contrast, $\log$ (VEGF) was significantly lower during pregnancy than the postpartum period (figure 2B, $\mathrm{p}<0.0001$, linear mixed model and Tukey multiple comparisons test). $\log (\mathrm{sFlt}-1)$ was significantly negatively associated with $\log$ (VEGF) throughout pregnancy $(r=$ $-0.92, \mathrm{p}<0.0001$, linear mixed model $). \log (\mathrm{sFlt}-1)$ was significantly associated with CCT $(\mathrm{p}=0.0037)$ but not with CVI ( $p=0.24$, linear mixed model). Since 29 of 48 VEGF values $(60.4 \%)$ were less than the lower limit of detection in the ELISA assay during pregnancy, the optimal model for the CCT or CVI was investigated using $\log (\mathrm{sFlt}-1)$.

As a result of AICc model selection, the optimal model for the CCT throughout pregnancy included age, AL, DBP and $\log (\mathrm{sFlt}-1) \quad(\mathrm{AICc}=938.4)$. In contrast, the optimal model for the CVI included only AL $(\mathrm{AICc}=469.7)$ (table 2).

\section{DISCUSSION}

In the current study, we analysed the temporal changes of the CCT and CVI and their associations with serum sFlt-1 during the pregnancy. Our results show that the CCT ratios were significantly higher in the second and third trimester of pregnancy than in the postpartum period; however, this was not the case with the CVI during pregnancy. Moreover, serum sFlt-1 was significantly associated with CCT during pregnancy. Taken together, sFlt-1 has a physiological effect on the choroidal structure during the pregnancy.

In general, choroidal vascular hyperpermeability is observed in eyes with CSC. ${ }^{5}$ Considering that pregnant women are prone to CSC, it was hypothesised that the CVI may gradually increase during the pregnancy. However, in this study, there were no significant changes in the CVI throughout pregnancy. Choroidal thickening in eyes with CSC may be accompanied by dilation of the choroidal vessels (increased CCT and CVI). However, our current results suggest that the changes in the choroidal structure throughout pregnancy were slightly different from that in eyes with CSC.

sFlt-1 is an endogenous antagonist of both VEGF and placental growth factor (PlGF). ${ }^{11}$ Previous reports have suggested that serum sFlt-1 was higher, whereas serum VEGF was lower, in pregnant women compared with nonpregnant women. ${ }^{12}{ }^{13}$ Our current results are consistent with those of previous studies, however, it was difficult to precisely explain the effect of sFlt- 1 on the choroidal structure during the pregnancy. Based on the physiological function of VEGF on vascular hyperpermeability, 

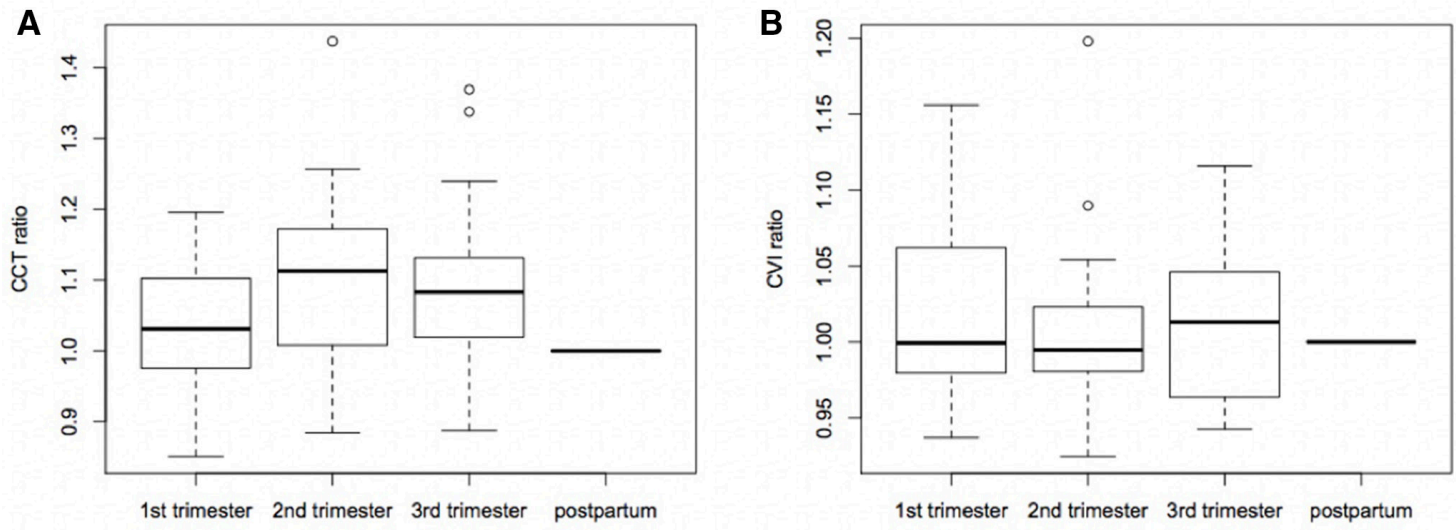

Figure 1 The changes of the central choroidal thickness (CCT) ratio and Choroidal Vascular Index (CVI) ratio in normal pregnant women $(A, B)$.

it was expected that low VEGF levels during pregnancy would result in reduced CCT and lower CVI. Future studies are needed to elucidate the association between high sFlt-1 and low VEGF levels, and thick CCT during the pregnancy.

Maternal serum sFlt-1 levels increase 5 weeks prior to the onset of pre-eclampsia; therefore, sFlt- 1 is considered a key factor in precipitating pre-eclampsia. ${ }^{14}$ Our previous study has suggested that the CVI was higher during the first trimester of pregnancy compared with healthy control participants, and that it was positively associated with SBP. ${ }^{10}$ However, this study revealed no significant association between the CVI and SBP throughout pregnancy. This discrepancy may be due to the fact that some participants with pre-eclampsia could not undergo OCT measurement and blood sampling in late pregnancy because of premature birth. This was a limitation of this study; therefore, a future study with a larger sample size is required to reveal the association between the CVI and SBP. PIGF is a member of the VEGF family and is clearly distinguishable from VEGF. In the obstetric field, there are previous reports indicating that both sFlt- 1 and PIGF

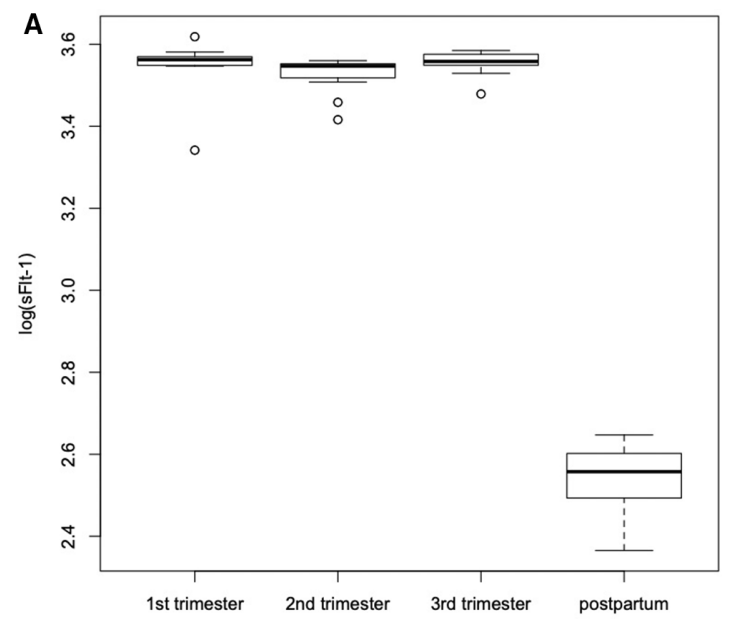

are involved in pre-eclampsia. ${ }^{14}{ }^{15}$ However, the serum PIGF was not measured in each participant; therefore, this was also one of the limitations of this study. Further investigation would be of interest to confirm whether or not PlGF is associated with the choroidal structure during the pregnancy.

Another limitation is a relatively small sample size in this study. However, a significant correlation between sFlt-1 and CCT throughout the pregnancy even in such a small sample size suggests a strong association between these two variables. A future study would be performed to confirm the current results, preparing a larger number of participants.

In summary, we found that CCT was significantly thicker in the second and third trimester of pregnancy, whereas CVI demonstrated no significant changes in healthy pregnant women. Moreover, serum sFlt-1 levels were associated with CCT during pregnancy, suggesting the possibility that OCT measurement may be useful for predicting the risk of not only CSC but also pre-eclampsia.

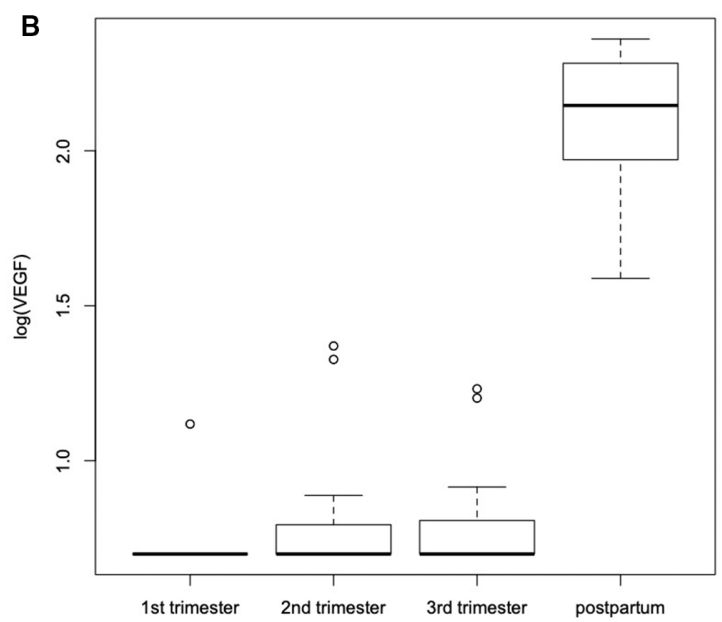

Figure 2 The temporal changes of serum soluble fms-like tyrosine kinase-1 (sFlt-1) and vascular endothelial growth factor (VEGF) in normal pregnant women (A, B). 
Table 2 The optimal model for the CCT and CVI during pregnancy

\begin{tabular}{|c|c|c|c|c|c|c|}
\hline & \multicolumn{3}{|c|}{ The optimal model for the CCT } & \multicolumn{3}{|c|}{ The optimal model for the CVI } \\
\hline & Coefficient & SE & $P$ value & Coefficient & SE & $P$ value \\
\hline Age & -12.1 & 3.80 & 0.0045 & N.S. & & \\
\hline$A L$ & -48.5 & 7.91 & $<0.0001$ & -0.38 & 0.23 & 0.11 \\
\hline SBP & N.S. & & & N.S. & & \\
\hline DBP & -0.94 & 0.36 & 0.011 & N.S. & & \\
\hline Log(sFlt-1) & 12.1 & 4.62 & 0.011 & N.S. & & \\
\hline
\end{tabular}

AL, axial length; CCT, central choroidal thickness; CVI, Choroidal Vascular Index; DBP, diastolic blood pressure; N.S., not selected; SBP, systolic blood pressure; sFlt-1, soluble fms-like tyrosine kinase-1.

Acknowledgements We would like to thank Editage (www.editage.com) for English language editing.

Contributors TS was involved in the study design, data collection, analysis of the results, and manuscript drafting. TI and KA participated in the study design, data collection, and manuscript reviewing and editing. $\mathrm{NI}, \mathrm{CA}, \mathrm{AO}, \mathrm{TN}, \mathrm{TI}, \mathrm{MM}-\mathrm{I}, \mathrm{YY}, \mathrm{KK}$ $\mathrm{RA}$ and RO participated in data collection and manuscript review and editing. All authors read and approved the final manuscript.

Funding The authors have not declared a specific grant for this research from any funding agency in the public, commercial or not-for-profit sectors.

Competing interests None declared.

Patient and public involvement Patients and/or the public were involved in the design, or conduct, or reporting, or dissemination plans of this research. Refer to the Methods section for further details.

Patient consent for publication Not applicable.

Ethics approval This prospective study was approved by the Institutional Review Board of the University of Tokyo (approval number: 11924).

Provenance and peer review Not commissioned; externally peer reviewed.

Data availability statement Data are available on reasonable request.

Open access This is an open access article distributed in accordance with the Creative Commons Attribution Non Commercial (CC BY-NC 4.0) license, which permits others to distribute, remix, adapt, build upon this work non-commercially, and license their derivative works on different terms, provided the original work is properly cited, appropriate credit is given, any changes made indicated, and the use is non-commercial. See: http://creativecommons.org/licenses/by-nc/4.0/.

ORCID iDs

Takafumi Suzuki http://orcid.org/0000-0002-7385-415X

Ryo Asaoka http://orcid.org/0000-0001-7182-1912

Ryo Obata http://orcid.org/0000-0002-1762-0797

\section{REFERENCES}

1 Ouzounian JG, Elkayam U. Physiologic changes during normal pregnancy and delivery. Cardiol Clin 2012;30:317-29.
2 Errera M-H, Kohly RP, da Cruz L. Pregnancy-associated retinal diseases and their management. Surv Ophthalmol 2013;58:127-42.

3 Park YJ, Park KH, Woo SJ. Clinical features of pregnancyassociated retinal and choroidal diseases causing acute visual disturbance. Korean J Ophthalmol 2017;31:320.

4 Chumbley LC, Frank RN. Central serous retinopathy and pregnancy. Am J Ophthalmol 1974;77:158-60.

5 Terao N, Koizumi H, Kojima K, et al. Association of upregulated angiogenic cytokines with choroidal abnormalities in chronic central serous chorioretinopathy. Invest Ophthalmol Vis Sci 2018;59:5924-31.

6 Pang L, Wei Z, Li O, et al. An increase in vascular endothelial growth factor (VEGF) and VEGF soluble receptor-1 (sFlt-1) are associated with early recurrent spontaneous abortion. PLoS One 2013;8:e75759

7 Yelumalai S, Muniandy S, Zawiah Omar S, et al. Pregnancy-induced hypertension and preeclampsia: levels of angiogenic factors in Malaysian women. J Clin Biochem Nutr 2010;47:191-7.

8 Sonoda S, Sakamoto T, Yamashita T, et al. Choroidal structure in normal eyes and after photodynamic therapy determined by binarization of optical coherence tomographic images. Invest Ophthalmol Vis Sci 2014;55:3893-9.

9 Sonoda S, Sakamoto T, Kuroiwa N, et al. Structural changes of inner and outer choroid in central serous chorioretinopathy determined by optical coherence tomography. PLoS One 2016;11:e0157190-16

10 Azuma K, Okubo A, Suzuki T. Assessment of choroidal structure in first trimester pregnant women. Sci Rep 2021;11:4629.

11 Roberts JM, Rajakumar A. Preeclampsia and soluble FMS-like tyrosine kinase 1. J Clin Endocrinol Metab 2009;94:2252-4.

12 Patrelli TS, Gizzo S, Plebani M, et al. The trend of VEGF-A and PIGF in pregnant patients: a perspective case-control study on 214 women. Clin Exp Obstet Gynecol 2012;39:57-64.

13 Cerdeira AS, Kandzija N, Pargmae P, et al. Circulating soluble FMSlike tyrosine kinase- 1 is placentally derived in normal pregnancy: first in vivo evidence. Pregnancy Hypertens 2019;16:145-7.

14 Levine RJ, Maynard SE, Qian C, et al. Circulating angiogenic factors and the risk of preeclampsia. N Engl J Med 2004;350:672-83.

15 Black C, Al-Amin A, Stolarek C, et al. Midpregnancy prediction of pre-eclampsia using serum biomarkers sFlt-1 and PIGF. Pregnancy Hypertens 2019;16:112-9. 